\title{
COVID-19 Infection and Myocardial Infarction Pathophysiology and Therapy
}

\author{
Authors: \\ Manraj Raja Singh Gill, *John A. Ambrose \\ Department of Cardiology, University of California, San Francisco, California, USA \\ *Correspondence to jamambrose@yahoo.com \\ Disclosure: \\ The authors have declared no conflicts of interest. \\ Received: \\ 10.12.20 \\ Accepted: \\ 30.04.21 \\ Keywords: \\ COVID-19, myocardial infarction (MI), thrombosis. \\ Citation: \\ EMJ Cardiol. 2021;9[1]:98-107.
}

\section{Abstract}

The relationship between COVID-19 and cardiovascular disease has been of interest since the beginning of the pandemic, with the focus more recently shifting towards thrombotic complications, including myocardial infarction (MI). While the inflammatory burden of infection has previously been implicated in the pathogenesis of $\mathrm{MI}$, at least early in the pandemic, many hospitals were seeing fewer ST-elevation MI admissions and the delivery of acute coronary syndrome care was disrupted in multiple ways. Furthermore, patients presenting with both COVID-19 infection and MI have been noted in small studies to have unique characteristics that pose clinical challenges, and there is reason to believe that standard therapy for both the prevention and treatment of all thrombotic events, including MI, may not be adequate. The aim of this article is to review the data regarding $\mathrm{Ml}$ and other thrombotic events during the pandemic, to explore the link between inflammation and thrombosis, and to suggest possible novel therapeutic options for the treatment and prevention of thrombosis in patients with COVID-19.

\section{INTRODUCTION}

There have been a number of concerns from the early stages of the COVID-19 pandemic regarding its implications in cardiovascular disease. Early on in the pandemic, standard acute coronary syndrome (ACS) care had been disrupted in several ways, and of particular interest in the recent literature was the relationship of COVID-19 to coronary artery disease and acute myocardial infarction (MI). This paper reviews the pandemic's effects on ACS care; discusses the overlapping pathophysiology between COVID-19 infection, $\mathrm{MI}$, and mimics of $\mathrm{Ml}$; and explores potential therapeutic strategies for treating and

preventing thrombotic complications in patients with COVID-19.

The interplay between infection and $\mathrm{MI}$ has been previously well-described, namely in the setting of influenza and bacterial pneumonias.' The prothrombotic and procoagulant state that is associated with acute infection further increases the risk of thrombosis, and this phenomenon has indeed been noted in the setting of advanced COVID-19 infection. A retrospective study of $>3,000$ hospitalised patients with COVID-19 in New York City, New York, USA, found an incidence of $\geq 1$ thrombotic event in $16 \%$ of patients, most of whom were on prophylactic-dose anticoagulation $(6.2 \%$ had venous thromboembolism and $11.1 \%$ had arterial, 
with $1.6 \%$ of those being ischaemic stroke and $8.9 \%$ being $\mathrm{MI}) ;^{2}$ however, the definition of $\mathrm{MI}$ was not specified in that report. Likewise, in a large registry from Boston, Massachusetts, USA, of $>1,100$ patients with COVID-19, arterial and venous thromboembolism occurred with high frequency in patients in the intensive care unit (ICU) (35.3\%), despite a high utilisation of thromboprophylaxis (>85\%). Symptomatic venous thromboembolism accounted for $27 \%$ of these events in the ICU. $\mathrm{Ml}$ occurred in $7.7 \%$, all of which were non-STelevation MI (NSTEMI) and possibly all Type 2. In hospitalised, non-ICU patients, the incidence of arterial and venous thromboembolism was $2.6 \%{ }^{3}$

\section{TRENDS IN MYOCARDIAL INFARCTION DURING THE PANDEMIC}

As COVID-19 spread across the world, disturbing trends were noted early on in ST-elevation $\mathrm{Ml}$ (STEMI) presentations and outcomes in some hospitals, and many oddities were noted in patients presenting with STEMI.

\section{Reductions in STEMI Incidence}

It is presently unclear whether STEMI is increased due to COVID-19 infection. To the contrary, some data have shown a reduction in STEMI presentations during the first months of the pandemic. Data compiled from 1,372 chest pain centres in China found a $26 \%$ reduction in STEMI presentations after the China Chest Pain Center's modified STEMI protocol was introduced on $23^{\text {rd }}$ January 2020 (that protocol being one that prioritised thrombolysis over primary percutaneous coronary intervention $[\mathrm{PCl}]) .{ }^{4} \mathrm{~A}$ similar trend was seen in some Italian and North American centres when COVID-19 was surging in those areas. ${ }^{5,6}$

\section{Presentation and Treatment Delays}

Along with this apparent decrease, delays in ACS care were frequently noted in registry data. From China, there was a numerical but insignificant increase in symptom-to-first-medical-contact time during the initial COVID-19 surge across their chest pain centre network of approximately 1 hour, on average. ${ }^{4}$ In Italy, the time from symptom onset to coronary angiography increased by $39.2 \%$ during the COVID-19 surge. ${ }^{5}$ Data from 75 hospitals in Spain noted similar findings. ${ }^{7}$

\section{Worsening Outcomes}

These trends are very concerning given the increased morbidity and mortality associated with STEMI treatment delays, and observational data have indeed noted increases in both during the pandemic. In China, in-hospital mortality increased from $4.6 \%$ to $7.3 \%$ and in-hospital heart failure increased from $14.2 \%$ to $18.4 \%$ during the outbreak period. ${ }^{4}$ In Italy, STEMI case fatality rates were more dramatically increased, at $13.7 \%$ versus the $4.1 \%$ registered in 2019 , and major complications were registered in $18.8 \%$ of cases in 2020 versus $10.4 \%$ in 2019. About $10 \%$ of patients in that registry were COVID19-positive, and the case fatality rate among COVID-19-positive STEMIs was substantially higher (28.6\%) compared with all other STEMI patients registered during the same week in 2020 (11.9\%). ${ }^{5}$ In the USA, the Providence St. Joseph Health system noted an observed-to-expected mortality ratio of 1.96 for patients with STEMI during the early stages of the pandemic. ${ }^{8}$

\section{Increased Incidence of Out-of-Hospital Cardiac Arrest}

The aforementioned observations beg the question: where have the STEMIs gone? It seems unlikely that MIs dramatically decreased during the pandemic, and various explanations have been offered, including patient avoidance of healthcare settings and misdiagnosis by providers in a time of crisis. Given the perceived hesitation of patients to present to healthcare settings during the pandemic, there is a deadlier possibility for this decrease. Delaying presentation with STEMI might increase out-of-hospital cardiac arrest due to unstable ventricular dysrhythmias. Global trends in out-of-hospital cardiac arrest support this hypothesis, with Italy seeing a 58\% increase during their surge ${ }^{9}$ and New York seeing a 5-fold increase. Furthermore, the proportion of those found dead on scene doubled compared to the same time period in 2019. ${ }^{10}$

It is presently unclear to what extent these aberrations in ACS presentations and care persist as the pandemic continues unabated and how the recent surges in COVID-19 infections seen worldwide will subsequently affect admission rates. Furthermore, it also remains unknown how pervasive these aberrations existed worldwide, even during the first months of the pandemic, 
as articles showing abnormalities were more likely published. However, a recent retrospective registry from 77 European centres assessing STEMI admissions early in the pandemic noted significant reductions in only $39 \%$ of centres, which was unrelated to COVID-19 incidence."

\section{STEMI IN PATIENTS WITH COVID-19 INFECTION}

\section{Absence of Culprit Lesions}

STEMI care during the pandemic was further complicated by the phenomenon of STEMI mimics identified in small studies. An early registry from Italy of 28 patients with COVID-19, all of whom met guideline definitions of STEMI at presentation, underwent coronary angiography, with $11 / 28$ found to not have a culprit lesion. Seven of these 11 had regional wall motion abnormalities on echocardiography. In patients without a culprit lesion, the investigators were unable to determine the aetiology of their clinical presentation. ${ }^{2}$

In another series from New York of 18 patients with COVID-19 who had ST-segment elevation (only one-third with a documented complaint of chest pain), a total of nine patients (50\%) underwent coronary angiography and only six of these patients (67\%) were noted to have obstructive disease. ${ }^{13}$ Since this publication, it has become apparent that myocarditis, cytokine-mediated myocardial injury, stress-induced cardiomyopathy, pulmonary embolism, and microvascular thrombosis are all clinical possibilities in patients with COVID-19 presenting with ischaemic symptoms and STelevations on ECG, with numerous case reports and series detailing cardiovascular magnetic resonance and autopsy findings of patients with similar presentations. ${ }^{14,15}$

There are differing opinions in the available literature regarding the incidence of myocarditis in patients with COVID-19. While there are a number of reports detailing cardiac MRI findings of late gadolinium enhancement suggestive of myocarditis, ${ }^{16}$ pathology data thus far have not suggested that viral infiltration of the myocardium is associated with myocyte necrosis and therefore have not corroborated these imaging findings. ${ }^{17,18}$ Rather than myocarditis as the primary entity, pathologic findings indicated microvascular thrombosis with resulting focal myocyte necrosis, which might explain the MRI findings..$^{18} \mathrm{~A}$ list of the potential causes of $\mathrm{MI}$ and ACS-like presentations (with reference to the fourth universal definition of $\mathrm{MI}$ ) in patients who tested positive for COVID-19 is contained in Figure $1 .^{19}$

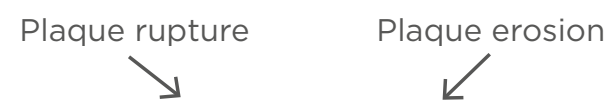

Type 1 myocardial infarction (Epicardial thrombosis)

Myocarditis

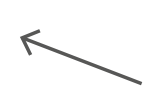

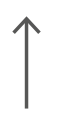

Potential causes for ACS and ACS-like presentations in COVID-19 infection embolism

Stress-induced cardiomyopathy

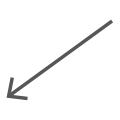

Type 2 myocardial infarction (Supply-demand mismatch)

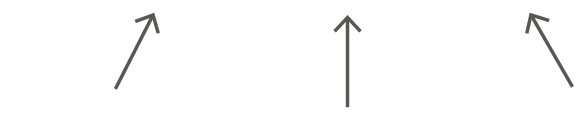

Hypoxia

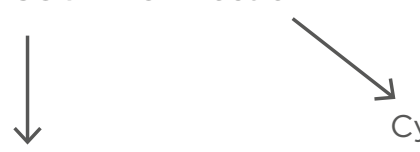

Cytokine-mediated myocardial injury
Microvascular thrombosis

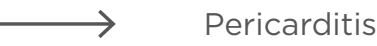

Pericarditis

Figure 1: Potential causes for acute coronary syndrome and acute coronary syndrome-like presentations in COVID-19 infection.

ACS: acute coronary syndrome. 


\section{Unique Features of Culprit Lesions}

In patients with COVID-19 and STEMI with confirmed obstructive disease, Choudry et al. ${ }^{20}$ assessed angiographic characteristics of patients who tested positive for COVID-19 versus patients who tested negative for COVID-19 during the same time period. This was a single-centre, observational study of 115 consecutive patients, and 39 (33.9\%) were diagnosed with concurrent COVID-19 infection. In those who were COVID-19 positive, there were significantly higher rates of multi-vessel thrombosis, stent thrombosis, and a higher thrombus burden with consequently higher use of GP Ilb/IIla inhibitors and thrombus aspiration versus COVID-19-negative STEMIs. Higher heparin doses to achieve therapeutic activated clotting times were also noted, and myocardial blush grade and left ventricular function were significantly lower with higher inhospital mortality. ${ }^{20}$ These observations support the hypothesis that COVID-19 may increase arterial thrombosis in epicardial coronary vessels and, with STEMI, a thrombotic burden that might be challenging to overcome with current therapies.

\section{COVID-19 INFECTION, NSTEMI, MYOCARDIAL INFARCTION TYPES 1 AND 2, AND MYOCARDIAL INJURY}

Data regarding COVID-19 and Type 1 NSTEMI are more difficult to dissect given the overlap with Type $2 \mathrm{Ml}$ and myocardial injury alone without MI. As in STEMI, it is unknown if NSTEMI (Types 1 and 2) occurrence is increased with COVID-19 infection or if its incidence is greater than that seen with other viruses. Myocardial injury (elevated troponin levels) is common in patients with COVID-19, with increasing frequency noted with increased disease severity, and up to $100 \%$ prevalence in small studies of those critically ill. ${ }^{21}$ Non-COVID-19 studies have demonstrated that myocardial injury is more likely to occur in critically ill, older patients and in those with comorbidities, ${ }^{20}$ with similar data reported in those infected with COVID-19.21 Furthermore, myocardial injury with concomitant echocardiographic abnormalities including left ventricular, right ventricular, and pericardial abnormalities portended a poorer prognosis than elevated troponin levels alone, suggesting echocardiography as a potential prognostic tool in evaluating the significance of myocardial injury in patients with COVID-19. ${ }^{22}$

The responses to acute infection, including the release of cytokines and catecholamines, as well as hypoxia, acidosis, tachycardia, and/or hypotension, are associated with Type 2 NSTEMI. While there appears to be a large proportion of patients with COVID-19 presenting with acute myocardial injury, NSTEMI rates have mirrored STEMI in their decline during the pandemic, ${ }^{5-7}$ raising the question of how many NSTEMIs (particularly Type 2 Mls) are being labelled as troponin elevation only (acute myocardial injury). Unfortunately, the criteria for diagnosis of Type 2 $\mathrm{Ml}$ are not specific and are variably interpreted, even in the absence of infection, making any Type 2 data difficult to interpret. ${ }^{23}$

\section{PATHOGENESIS OF THROMBOSIS IN THE SETTING OF COVID-19 INFECTION}

The interplay between endothelial injury, inflammation, and thrombosis has been longrecognised, and COVID-19 infection is implicated in these processes. Increases in proinflammatory cytokines such as IL-1 and TNF-a lead to an imbalance that promotes thrombosis in various vascular beds. An amplification loop in cytokine production (cytokine storm) promotes a prothrombotic milieu potentially leading to venous, microvascular, as well as large-vessel thrombosis causing $\mathrm{MI}$ and stroke. The endothelial cell lining of all vascular beds is a prime target of the virus, and viral penetration into the endothelium along with proinflammatory cytokines and other molecules change the normally protective antithrombotic, anti-inflammatory, vasodilatory endothelium into an altered proinflammatory, prothrombotic, and vasoconstrictor substrate. ${ }^{24-26}$ Certain conditions such as diabetes, obesity, and the metabolic syndrome can further potentiate these processes, and the presence of angiotensinconverting enzyme 2 receptors on adipose tissue may provide additional reason for poorer prognosis in patients with these conditions. ${ }^{27,28}$ In patients with underlying coronary artery disease, the proinflammatory, prothrombotic milieu of COVID-19 infection may promote destabilisation of lipid-rich plaques leading to rupture and thrombus, the usual substrate for 
STEMI. Acute phase reactants such as fibrinogen, plasminogen activator inhibitor-1, and C-reactive protein potentiate this prothrombotic and proinflammatory state and serve as biomarkers of inflammation in COVID-19 infections.

This prothrombotic milieu is also associated with the observation that the fibrin degradation product D-dimer appears to be a strong prognostic marker associated with high mortality in patients with COVID-19, with an early study from China noting that D-dimer level of $>2.14 \mathrm{mg} / \mathrm{L}$ predicted in-hospital mortality with a sensitivity of $88.2 \%$ and specificity of $71.3 \%$ in a series of 250 patients. ${ }^{29}$ More recent observational studies of larger patient populations have also noted the strength of this association, further suggesting that D-dimer serves as a reliable marker of prognosis. ${ }^{30}$ In the observational data from New York that found high thrombosis rates in patients hospitalised with COVID-19, all-cause mortality was notably higher in those with thrombotic events, at $43.2 \%$ versus $21.0 \%$ without thrombosis. ${ }^{2}$

Disseminated intravascular coagulopathy (DIC) or a DIC-like picture is common in patients critically ill and dying from COVID-19. ${ }^{31}$ However, in the latter, it is hypothesised that some patients develop organ failure caused by thrombi in micro-vessels with some features of DIC. Unlike classic DIC, however, there are findings of elevated fibrinogen levels, only moderately low platelets, and little evidence of a bleeding diathesis. This picture is more consistent with an immune-triggered, complement-mediated thrombotic microangiopathy, ${ }^{32}$ which would require different therapies than classic DIC. As alluded to earlier, pathology data also noted that microvascular thrombi found at autopsy in patients who died of COVID-19 infection contained higher concentrations of fibrin and complement components than in COVID-19negative controls. ${ }^{18}$ This phenomenon of fibrinrich microvascular thrombi may be an example of this thrombotic microangiopathy and could also contribute to elevated D-dimer levels in patients critically ill with COVID-19 without evidence of large-vessel thrombosis. These microvascular thrombi might also explain the patchy late gadolinium enhancement found on cardiac MRI.

\section{POTENTIAL THERAPEUTIC APPROACHES}

Given the mortality associated with thrombotic complications, preventing and treating inflammation-driven thrombosis is paramount. The remainder of this paper discusses potential therapeutic approaches that warrant further investigation.

\section{Patients with COVID-19 Presenting with Acute Coronary Syndrome}

Controversy remains surrounding ACS care since the pandemic onset, starting with the Chinese Chest Pain Center network prioritising thrombolysis in STEMI as the preferred treatment strategy. However, morbidity and mortality increased in patients with STEMI after protocol introduction. ${ }^{4}$

The joint statement of the Society for Cardiovascular Angiography and Interventions (SCAI), American College of Cardiology (ACC), and American College of Emergency Physicians (ACEP) stressed that primary $\mathrm{PCl}$ is still the standard reperfusion strategy in patients with STEMI with suspected COVID-19 infection, particularly in those with high-risk features. Their algorithm allows for bedside echocardiography to confirm regional wall motion abnormalities prior to cardiac catheterisation laboratory activation, especially if there are equivocal features in presentation. ${ }^{33}$ Coronary CT may be preferable initially to invasive angiography in equivocal cases to limit hospital staff exposure. ${ }^{34}$ In cases of patients with suspected COVID-19 and STEMI presenting to non-PCl centres with low likelihood of immediate transfer for $\mathrm{PCl}$, thrombolytic therapy could be considered as long as high-risk features are absent. ${ }^{35}$ However, as noted earlier, some COVID-positive STEMI-like presentations, even with regional wall motion abnormalities on echocardiography, do not have angiographic culprit lesions. This calls into question the utility of thrombolytic therapy even with prior echocardiography. A proposed approach is outlined in Figure 2.

New recommendations for pharmacotherapy in patients with COVID-19 and STEMI have not been made. Patients with COVID-19 and STEMI are unique and may have a large thrombotic burden and worse outcomes. ${ }^{12,20}$ Is standard dual 
Possible STEMI

COVID-19 positive or suspected

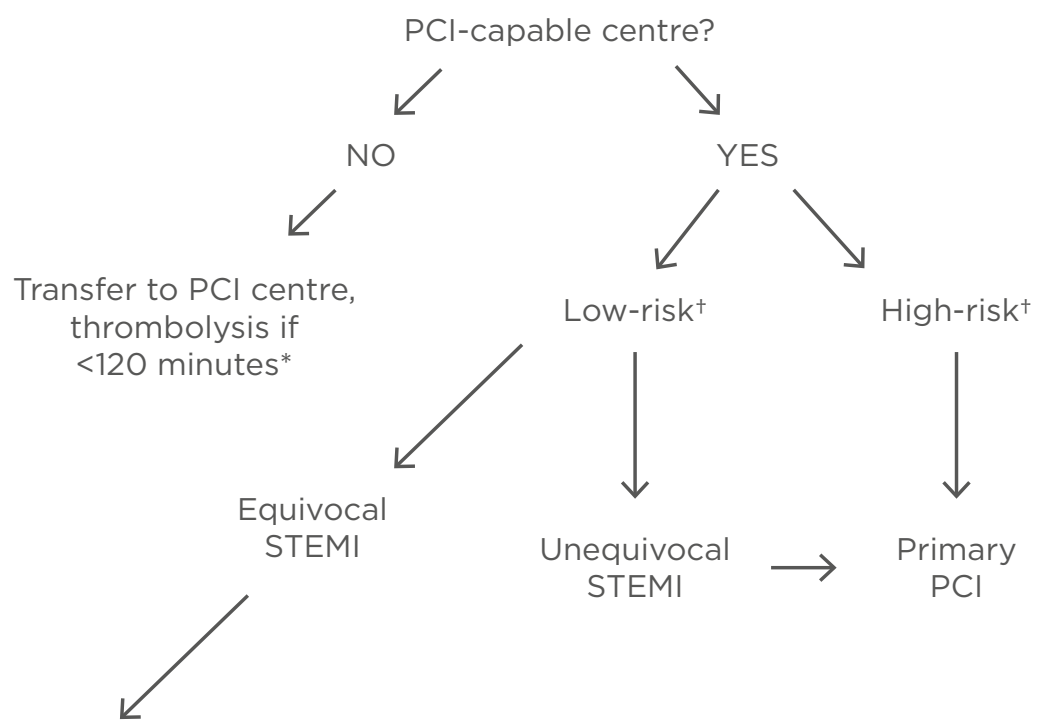

Consider coronary CT

angiogram $\ddagger$

Figure 2: Potential ST-elevation myocardial infarction treatment algorithm.

*Time from presentation at non-PCI hospital to estimated balloon time at $\mathrm{PCl}$ centre. Thrombolytic therapy to be administered only if unequivocal STEMI.

${ }^{+}$Risk assessment per Killip class, infarct-related artery, presence or absence of haemodynamic or electrical instability, timing of presentation.

‡To define presence of infarct-related lesion amenable to $\mathrm{PCl}$.

PCl: percutaneous coronary intervention; STEMI: ST-elevation myocardial infarction.

antiplatelet therapy and systemic anticoagulation enough? This approach may need to be tailored, especially in those critically ill with seemingly insurmountable inflammation-born thrombosis. Higher doses of heparin were required in the London, UK, registry to achieve therapeutic activated clotting times. ${ }^{20}$ Given the outcomes for those in the COVID-19 STEMI group, as noted previously, a more aggressive pharmacotherapy may be warranted; however, at the present time, the optimal approach remains unclear.

What about COVID-19 patients with STEMI/ NSTEMI-like presentations without culprit lesions? The differential includes stressinduced cardiomyopathy, pulmonary embolism, microvascular thrombosis, and myocarditis. In NSTEMI, the current recommendation is for early/ urgent angiography only with high-risk clinical features, haemodynamic instability, or a Global Registry of Acute Coronary Events (GRACE) score exceeding $140 .{ }^{33}$ NSTEMI care in patients who test positive for COVID-19 is confounded by observer variability regarding the diagnosis of Type 1 versus Type 2 NSTEMI or myocardial injury. Therefore, astute clinical diagnosis is needed prior to committing to a treatment plan. It appears that most NSTEMI are Type 2, which appears similar to what is found in patients who are seriously ill without COVID-19 in an ICU setting and is usually managed by treating the underlying condition.

\section{Preventing Thrombosis in COVID-19 Infection}

Can thrombotic complications in advanced COVID-19 infection be prevented, and who should receive these additional therapies? Breakthrough thrombotic events are common in patients hospitalised with COVID-19 who are receiving prophylactic anticoagulation, and are associated with poorer outcomes as mentioned earlier. A mortality benefit in patients receiving full-dose 


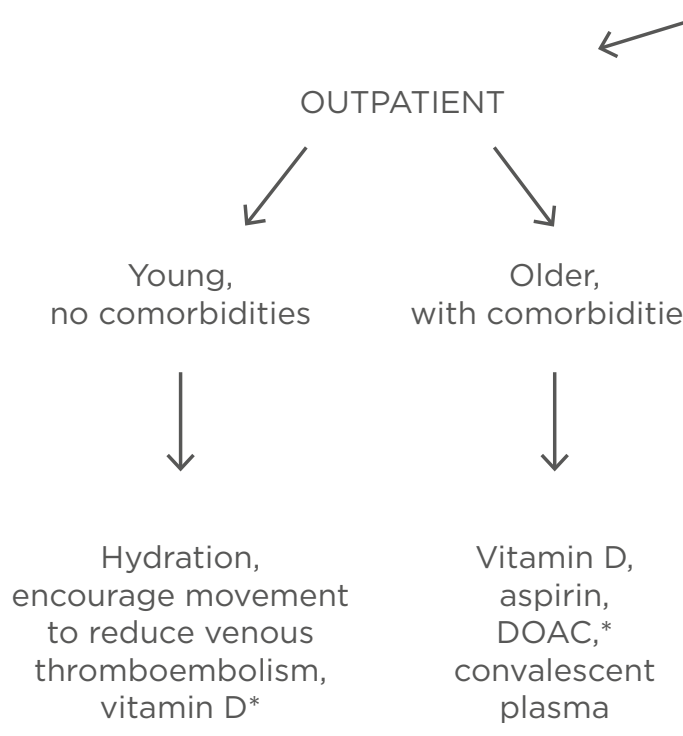

COVID-19 positive
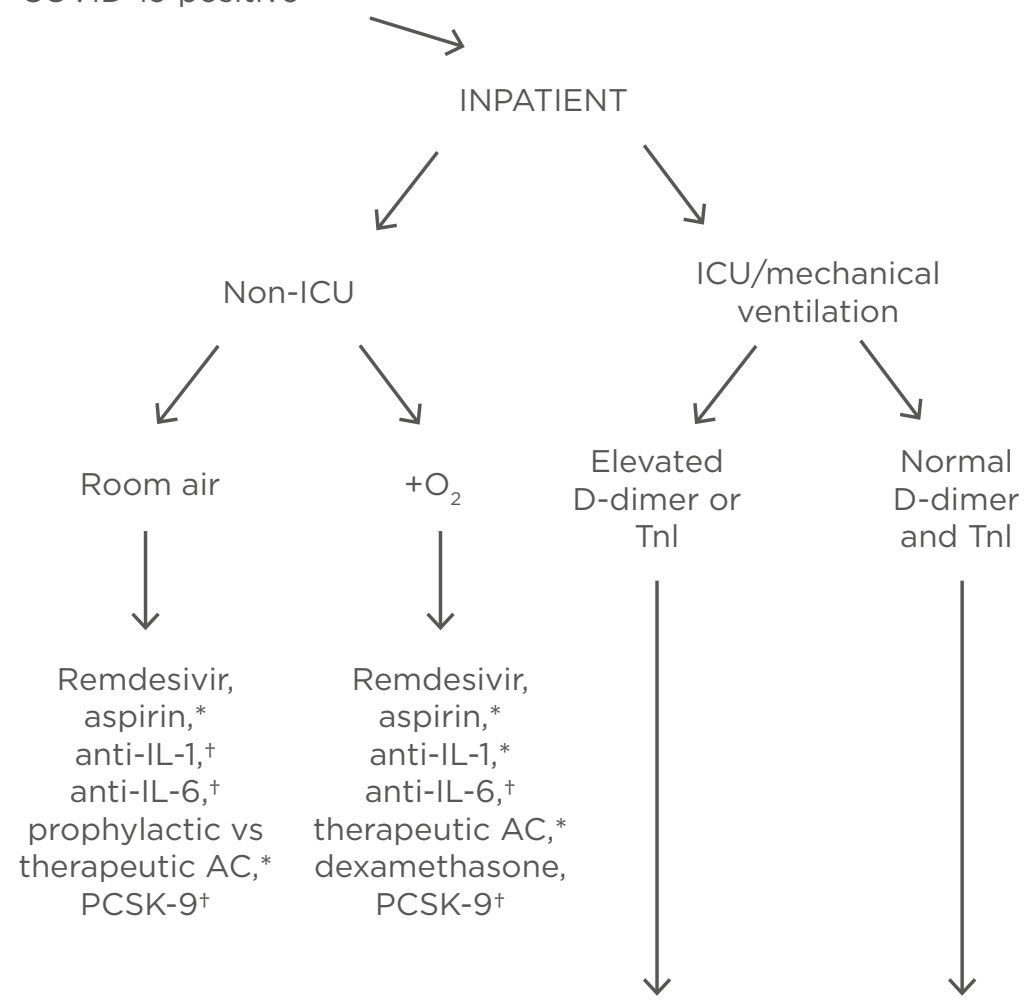

Aspirin,*

AC: prophylactic vs therapeutic, ${ }^{*}$ dexamethasone
Aspirin,* AC: prophylactic vs therapeutic, ${ }^{*}$ dexamethasone

\section{Figure 3: Potential thrombosis prevention algorithm.}

*Potential options, pending outcomes of ongoing randomised controlled trials.

+Hypothesised therapies warranting further investigation.

AC: anticoagulation; DOAC: direct oral anticoagulants; ICU: intensive care unit; PCSK-9: proprotein convertase subtilisin/kexin type 9; Tnl: troponin; vs: versus.

anticoagulation was noted by a retrospective observational study of 2,773 hospitalised patients with COVID-19. ${ }^{36}$ In those requiring mechanical ventilation, there was a significant mortality benefit with full-dose anticoagulation, with an in-hospital mortality of $29.1 \%$ versus $62.7 \%$ in those not receiving treatment-dose anticoagulation, suggesting that thrombotic phenomena are more likely to occur with advanced illness. Bleeding rates were naturally increased in the anticoagulation group. These observational data suggested that an approach of increased anticoagulation dosing with increased disease severity may be reasonable, but more data are needed.

Since initial publication, three collaborative randomised controlled trials investigating anticoagulation in patients hospitalised with COVID-19 (REMAP-CAP, ACTIV-4, and ATTACC) have halted enrolment of critically ill patients, as full-dose anticoagulation appeared to be futile in these patients and heightened bleeding complications. However, preliminary, unpublished data thus far have reportedly found benefits in moderately ill hospitalised patients, suggesting early initiation of therapy may be of importance. ${ }^{37}$ Other questions currently being explored are the utility of anticoagulation after discharge from the ICU, and whether oral anticoagulants at reduced doses have a role in patients with moderate illness severity.

What about patients with COVID-19 who have not been admitted to the hospital? As their symptoms are mild, do they need additional 
therapies to reduce thrombotic events? In the Boston study quoted earlier, ${ }^{3}$ there were no arterial or venous thromboembolic events in the 715 non-hospitalised patients. However, this was a younger population with fewer risk factors than those hospitalised. It is presently unknown whether some older patients not hospitalised with more comorbidities require a more aggressive antithrombotic strategy and, at the present time, no randomised data regarding this question have been published.

Beyond anticoagulation, can the current understanding of pharmacotherapy for the prevention and treatment of thrombotic events, including STEMI or Type 1 NSTEMI, be applied to mitigate these effects in severe COVID-19 infection? Inflammation can beget local thrombosis, and thrombosis can amplify inflammation. ${ }^{25}$ Is there a role for the prophylactic use of aspirin, an agent with both antiinflammatory and antithrombotic properties? Aspirin therapy in the setting of sepsis and acute respiratory distress syndrome has suggested a mortality benefit in observational studies. ${ }^{38}$ Several outpatient COVID-19-positive trials are presently underway, such as the PEAC trial and the LEAD COVID-19 trial, as well as an inpatient study lead by the RECOVERY group. However, no results of these studies are presently available.

Statins have been of interest given their pleiotropic effects, with initial reports suggesting lower mortality in patients with COVID-19 on statin therapy. However, these were observational reports with a likely selection bias for non-critically ill patients. Although statin use may decrease cytokine production in the setting of sepsis, ${ }^{39}$ previous trials and meta analyses in sepsis and ventilator-associated pneumonias have not shown a mortality benefit. ${ }^{40,41}$ Proprotein convertase subtilisin/kexin type 9 inhibitors are also of potential interest (no randomised data as of yet) given that experimental models have suggested that their effects are not limited to only lowering cholesterol (which itself may confer protection against viral entry in human cells), but also can improve endothelial function, reduce oxidative stress and platelet adhesion, increase stability of atherosclerotic plaques, and increase interferon- $\beta$ production. ${ }^{42}$

IL-1 inhibition with anakinra has been investigated in two small European studies, ${ }^{43,44}$ with findings of less frequent need for ICU transfer, decreased inflammatory markers, and improved mortality. Thrombotic outcomes were, however, not reported in these studies, and bacteraemia was predictably higher in those receiving immunosuppressive therapy. Tocilizumab, an IL-6 inhibitor, has recently been investigated in patients hospitalised with COVID-19. While no mortality benefit was noted in a randomised trial, subgroup analysis of non-critically ill patients initiated on this therapy were less frequently transferred to the ICU level of care than those in the placebo group, suggesting a possible benefit if initiated in the setting of non-severe illness. ${ }^{45}$

Colchicine is readily available, inexpensive, and has potent anti-inflammatory effects, with an already promising role in the treatment of coronary artery disease. ${ }^{46} \mathrm{~A}$ randomised and currently unpublished trial investigating the role for low-dose colchicine in treating COVID-19 infection reportedly did not suggest a significant clinical benefit in non-hospitalised patients, ${ }^{47}$ and the RECOVERY trial halted randomisation of inpatients to colchicine therapy due to an apparent lack of efficacy.

Dexamethasone therapy has become standard therapy in COVID-19 infection as it has demonstrated mortality benefit in patients requiring supplemental oxygen. However, the early results of the dexamethasone trial have not, as of yet, reported the incidence of thrombosis or markers of inflammation and thrombosis, ${ }^{48}$ and prior data on glucocorticoid administration and thrombosis are conflicting. Steroid administration has been implicated in thromboembolic events, ${ }^{49}$ yet, in inflammatory states, steroid administration reduces fibrinogen and von Willebrand factor, and increases plasminogen activator inhibitor-1. ${ }^{50}$ As decreasing the inflammatory burden of COVID-19 infection should decrease thrombotic complications and thrombotic events, this should be assessed in the thousands now receiving dexamethasone.

Vitamin $\mathrm{D}$ deficiency has been associated with venous thrombosis and $\mathrm{MI}$, and approximately $60 \%$ of Americans are deficient. ${ }^{51}$ However, the strength and mechanism of this association remains vague. In patients who are non-obese and vitamin D deficient without cardiovascular disease, supplementation with high-dose calcifediol reduced in vitro thrombin generation 
and clot density. ${ }^{52}$ A Spanish study randomised patients admitted with COVID-19 infection to high-dose calcifediol plus standard care versus standard care alone and found a significant reduction in the need for ICU care with calcifediol. ${ }^{53}$ However, at this time, data are mostly observational and uncontrolled. It remains unclear if vitamin $D$ supplementation in COVID-19 could potentially reduce thrombotic complications such as MI. See Figure 3 for a proposed algorithm for the prevention of thrombosis in COVID-19 infection.

\section{CONCLUSIONS}

COVID-19 infection can result in thrombosis in various vascular beds. While most thrombotic events are venous in origin, $\mathrm{Ml}$ is one of its manifestations and can manifest as STEMI or NSTEMI. While it is presently unclear if MIs related to thrombotic events in the epicardial coronary arteries are necessarily increased with COVID-19 infection, troponin elevations are common. The astute clinician must diagnose with all the available information whether the clinical syndrome represents either STEMI, NSTEMI Type 1, Type $2 \mathrm{MI}$, acute myocardial injury, one of the other MI mimics, or pulmonary embolism, and treat accordingly.

The authors believe that a bigger challenge is in the prevention of thrombotic complications in COVID-19 infection. While this paper has reviewed the data and offered some suggestions, further study is required; the data are rapidly evolving, with multiple new studies published weekly. Ongoing and future randomised trials or registries should provide important answers to some of these questions that could hopefully improve future outcomes. The ultimate solution to thrombosis is, of course, effective vaccines to prevent COVID-19 infection.

\section{References}

1. Musher D et al. Acute infection and myocardial infarction. $N$ Engl J Med. 2019;380(2):171-6.

2. Bilaloglu $S$ et al. Thrombosis in hospitalized patients with COVID-19 in a New York City health system. JAMA. 2020;324(8):799-801.

3. Piazza $\mathrm{G}$ et al. Registry of arterial and venous thromboembolic complications in patients with COVID-19. J Am Coll Cardiol. 2020;76(18):2060-72.

4. Xiang $D$ et al. Management and outcomes of patients with STEMI during the COVID-19 pandemic in China. J Am Coll Cardiol. 2020;76(11):1318-24

5. De Rosa $S$ et al. Reduction of hospitalizations for myocardial infarction in Italy in the COVID-19 era. Eur Heart J. 2020;41(22):2083-8.

6. Garcia S et al. Reduction in ST-segment elevation cardiac catheterization laboratory activations in the United States during COVID-19 pandemic. J Am Coll Cardiol. 2020;75(22):2871-2

7. Rodriguez-Leor $\mathrm{O}$ et al. Impact of COVID-19 on ST-segment elevation myocardial infarction care. The Spanish experience. Revista Espanola de Cardiologia. 2020;73(12):9941002.

8. Gluckman T et al. Case rates, treatment approaches, and outcomes in acute myocardial infarction during the Coronavirus disease 2019 pandemic. JAMA Cardiol. 2020;5(12):1419-24.

9. Baldi E et al. Out-of-hospital cardiac arrest during the Covid-19 outbreak in Italy. N Engl J Med. 2020;383:496-8.

10. Mountantonakis S et al. Out-ofhospital cardiac arrest and acute coronary syndrome hospitalizations during the COVID-19 surge. J Am Coll Cardiol. 2020;76(10):1271-3.

11. De Luca G et al. Impact of COVID-19 pandemic on mechanical reperfusion for patients with STEMI. J Am Coll Cardiol. 2020;76(20):2321-30.

12. Stefanini $\mathrm{G}$ et al. ST-elevation myocardial infarction in patients with COVID-19. Circulation. 2020;141(25):2113-6.

13. Bangalore $\mathrm{S}$ et al. ST-segment elevation in patients with COVID-19. N Engl J Med. 2020;382(25):2478-80.

14. Esposito A et al. Cardiac magnetic resonance characterization of myocarditis-like acute cardiac syndrome in COVID-19. J Am Coll Cardiol Img. 2020;13(11):2462-5.

15. Guagliumi G et al. Microthrombi and ST-segment elevation myocardial infarction in COVID-19. Circulation. 2020;142:804-9.

16. Kotecha T et al. Patterns of myocardial injury in recovered troponin-positive COVID-19

patients assessed by cardiovascular magnetic resonance. Eur Heart J. 2021;42(19):1866-78

17. Lindner $D$ et al. Association of cardiac infection with SARS-CoV-2 in confirmed COVID-19 autopsy cases. JAMA Cardiol. 2020;5(11):1281-5.

18. Pellegrini et al. Microthrombi as a major cause of cardiac injury in COVID-19 - a pathologic study. Circulation. 2021;143:1031-42.

19. Thygesen $\mathrm{K}$ et al. Fourth universal definition of myocardial infarction. J Am Coll Cardiol. 2018;72(18):2231-64.

20. Choudry F et al. High thrombus burden in patients with COVID-19 presenting with ST-segment elevation myocardial infarction. J Am Coll Cardiol. 2020;76(10):1168-76.

21. Sandoval $Y$ et al. Cardiac troponin for assessment of myocardial injury in COVID-19. J Am Coll Cardiol. 2020;76(10):1244-58.

22. Giustino $\mathrm{G}$ et al. Characterization of myocardial injury in patients with COVID-19. J Am Coll Cardiol. 2020;76(18):2043-55.

23. Saleh $\mathrm{M}$ et al. Misdiagnosis of type II myocardial infarction. J Am Coll Cardiol. 2019;74(13):1732-3.

24. Ambrose J, Bhullar A. Inflammation and thrombosis in coronary atherosclerosis: pathophysiologic 
mechanisms and clinical correlations. EMJ. 2019;4(1):71-8.

25. Libby P, Simon D. Inflammation and thrombosis - the clot thickens. Circulation. 2001;103(13):1718-20.

26. Libby P, Luscher T. COVID-19 is, in the end, an endothelial disease. Eur Heart J. 2020;41(32):3038-44

27. Sanchis-Gomar F et al. Obesity and outcomes in COVID-19: when an epidemic and pandemic collide. Mayo Clin Proc. 2020;95(7):1445-53.

28. Sharma A et al. Association of obesity with more critical illness in COVID-19. Mayo Clin Proc. 2020;95(9):2040-2

29. Yao $Y$ et al. D-dimer as a biomarker for disease severity and mortality in COVID-19 patients: a case control study. J Intensive Care. 2020;8:49.

30. He $X$ et al. The poor prognosis and influencing factors of high D-dimer levels for COVID-19 patients. Scientific Reports. 2021;11(1):1830.

31. Liao D et al. Haematological characteristics and risk factors in the classification and prognosis evaluation of COVID-19: a retrospective cohort study. Lancet Haematol. 2020;7(9):E671-8.

32. Merril JT et al. Emerging evidence of a COVID-19 thrombotic syndrome has treatment implications. Nat Rev Rheumatol. 2020;16:581-9.

33. Mahmud E et al. Management of acute myocardial infarction during the COVID-19 pandemic. J Am Coll Cardiol. 2020;76(11):1375-84

34. Rudski L et al. Multimodality imaging in evaluation of cardiovascular complications in patients with COVID-19. J Am Coll Cardiol. 2020;76(11):1345-57.

35. Daniels $M$ et al. Reperfusion of ST-segment-elevation myocardial infarction in the COVID-19 era. Circulation. 2020;141:1948-50.
36. Paranjpe I et al. Association of treatment dose anticoagulation with in-hospital survival among hospitalized patients with COVID-19. J Am Coll Cardiol. 2020;76(1):122-4.

37. NIH News Release. Full-dose blood thinners decreased need for life support and improved outcome in hospitalized COVID-19 patients. 2021. Available at: https://www.nih.gov/ news-events/news-releases/full-doseblood-thinners-decreased-needlife-support-improved-outcomehospitalized-covid-19-patients. Last accessed: 6 May 2021.

38. Toner $\mathrm{P}$ et al. Aspirin as a potentia treatment in sepsis or acute respiratory distress syndrome. Crit Care. 2015:19:374.

39. Novack $V$ et al. The effects of statin therapy on inflammatory cytokines in patients with bacterial infections: a randomized double-blind placebo controlled clinical trial. Intensive Care Med. 2009;35(7):1255-60.

40. Papazian L et al. STATIN-VAP study group effect of statin therapy on mortality in patients with ventilatorassociated pneumonia: a randomized clinical trial. JAMA. 2013;310(16):1692700

41. Pertzov B et al.

Hydroxymethylglutaryl-CoA reductase inhibitors (statins) for the treatment of sepsis in adults - a systematic review and meta-analysis. Clin Microbiol Infect. 2019;25:280-9.

42. Barkas F et al. Statins and PCSK9 inhibitors: what is their role in coronavirus disease 2019? Med Hypotheses. 2021;146:110452.

43. Huet $T$ et al. Anakinra for severe forms of COVID-19: a cohort study. The Lancet Rheumatology. 2020;2(7):e393-400.

44. Cavalli $\mathrm{G}$ et al. Interleukin-1 blockade with high-dose anakinra in patients with COVID-19, acute respiratory distress syndrome, and hyperinflammation: a retrospective cohort study. Lancet Rheumatol. 2020;2(6):e325-31.

45. Rosas I, et al. Tocilizumab in hospitalized patients with severe Covid-19 pneumonia. N Engl J Med. 2021;384:1491-502.

46. Tardif JC et al. Efficacy and safety of low-dose colchicine after myocardial infarction. $\mathrm{N}$ Engl J Med. 2019;381:2497-505.

47. Tardif JC, et al. Efficacy of colchicine in non-hospitalized patients with COVID-19. medRxiv. 2021;DOI:10.1101/ 2021.01.26.21250494

48. The RECOVERY Collaborative Group. Dexamethasone in hospitalized patients with Covid-19. N Engl J Med. 2021;384:693-704

49. Johannesdottir S et al. Use of glucocorticoids and risk of venous thromboembolism: a nationwide population-based case-control study. JAMA Intern Med. 2013;173(9):743-52.

50. Van Zaane B et al. Systematic review on the effect of glucocorticoid use on procoagulant, anti-coagulant and fibrinolytic factors. J Thromb Haemost. 2010;8(11):2483-93.

51. Khademvatani $\mathrm{K}$ et al. The relationship between vitamin D status and idiopathic lower-extremity deep vein thrombosis. Int J Gen Med. 2014;7:303-9.

52. Blondon $M$ et al. Thrombin generation and fibrin clot structure after vitamin D supplementation. Endocrine Connections. 2019;8(11):1447-54.

53. Castillo $M$ et al. Effect of calcifediol treatment and best available therapy versus best available therapy on intensive care unit admission and mortality among patients hospitalized for COVID-19: a pilot randomized clinical study. J Steroid Biochem Mol Biol. 2020;203:105751. 